\title{
Episode of Care Payment Method
}

National Cancer Institute

\section{Source}

National Cancer Institute. Episode of Care Payment Method. NCI Thesaurus. Code C70682.

The payment mechanism that is used to compensate a healthcare provider for the care provided to a patient in form of a fee for all services rendered during a single episode of care, such as a case that includes all components of care from the initial diagnosis of a condition to completed treatment and rehabilitation. 